\title{
Un feuillet d'incunable, ou le jeu des sept différences
}

\section{Daniel Bornemann}

\section{(2) OpenEdition}

1 Journals

Édition électronique

URL : https://journals.openedition.org/rbnu/2735

DOI : 10.4000/rbnu.2735

ISSN : 2679-6104

Éditeur

Bibliothèque nationale et universitaire de Strasbourg

\section{Édition imprimée}

Date de publication : 1 novembre 2012

Pagination : 68-71

ISSN : 2109-2761

\section{Référence électronique}

Daniel Bornemann, «Un feuillet d'incunable, ou le jeu des sept différences », La Revue de la BNU [En ligne], 6 | 2012, mis en ligne le 01 novembre 2012, consulté le 08 août 2021. URL : http:// journals.openedition.org/rbnu/2735; DOI : https://doi.org/10.4000/rbnu.2735

\section{(c) (i) (9)}

La Revue de la BNU est mise à disposition selon les termes de la Licence Creative Commons Attribution - Pas d'Utilisation Commerciale - Partage dans les Mêmes Conditions 4.0 International. 


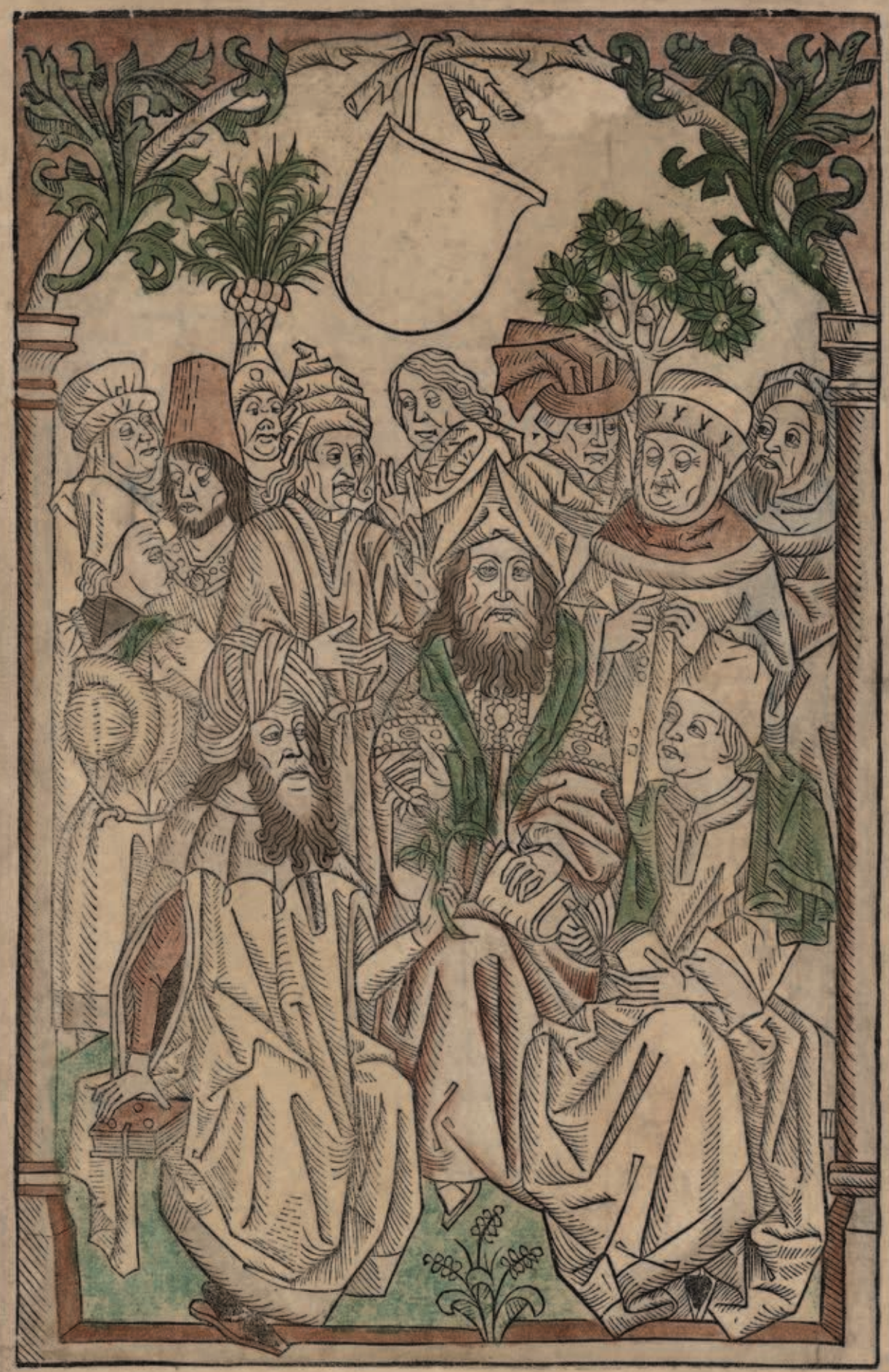

Verso du feuillet liminaire du Gart der Gesundheit ou Hortus sanitatis, de Johannes de Cuba, Strasbourg : Johann Grüninger, c. 1485-1486 (coll. BNU) 


\section{UN FEUILLET D'INCUNABLE, OU LE JEU DES SEPT DIFFÉRENGES}

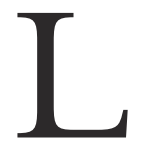

a BNU a pu acquérir en 2011 un feuillet isolé provenant d'un incunable alsacien : l'édition du Gart der Gesundheit (Hortus sanitatis ou "Jardin de santé") réalisée par l'imprimeur Johann Grüninger, sans doute en 1486. Ce feuillet a été retranché d'un volume, comme cela s'est beaucoup pratiqué à des époques heureusement révolues, où l'intégrité des incunables passait parfois derrière les pratiques commerciales. Les conservateurs condamnent ce procédé, mais conservent néanmoins pieusement dans leurs collections des exemples nombreux de telles pratiques, qui parfois représentent tout de même des restes intéressants pour l'érudition. C'est le cas pour ce feuillet liminaire d'un incunable, par ailleurs répertorié dans d'autres bibliothèques... dont certains exemplaires justement sont privés de leur premier feuillet. A la BNU, deux des éditions de ce même titre (l'une parue à Augsbourg en 1486 et l'autre, " sans lieu ni date ", identifiée comme étant celle de Bâle, parue chez Michael Furter, en 1486 environ) sont également privées de leurs premiers feuillets. La bibliothèque, qui ne possède pas cette édition de Grüninger, a décidé d'en acquérir au moins ce fragment, significatif dans l'histoire de la transmission des images dans les tout premiers livres illustrés.

L'auteur de ce livre de botanique et d'autres sciences naturelles appliquées à la médecine est le médecin ("Stadtarzt ") francfortois Johannes de Cuba, ce qui est la latinisation de Johann Wonnecke von Kaub. Son ouvrage compte comme le tout premier livre imprimé concernant les sciences naturelles. Il a été écrit en allemand ; les traductions latine et française datent respectivement de 1491 et 1500 (à Paris, chez Antoine Vérard). Mais l'histoire éditoriale de ce livre est riche et complexe.
Il a tout d'abord été imprimé par Peter Schoeffer, le successeur direct de Gutenberg, à Mayence, en 1485 (la date au colophon est le 28 mars 1485) et ses pages comportent 41 lignes. L'incunable, comme c'est en général le cas, n'a pas de page de titre. La première page de celui-ci, le premier recto, est laissée vierge de toute inscription, mais sur son revers, le premier verso, figure une gravure représentant une assemblée de treize médecins. Elle fait donc face à l'incipit du texte, en position de frontispice, et constitue une sorte de caution scientifique figurée donnée au seuil même du livre par cette assemblée. Les médecins ou pharmaciens ne sont pas identifiés, mais certains sont typés : médecins de l'Antiquité, médecins orientaux... Les identifier serait sans doute partiellement possible, avec beaucoup d'érudition. Galien, Avicenne, Serapion, Dioscoride, l'auteur des Pandectes Matthaeus Sylvaticus, Platearius rentrent en ligne de compte. Une édition strasbourgeoise bien plus tardive de l'herbier - ou de la flore (en allemand Kräuterbuch) - de Brunfels, paru en 1539 chez $\mathrm{H}$. Schott, représente également, mais chacun dans un compartiment séparé, une série de médecins et de pharmaciens, en les nommant. On pourra sans doute y retrouver quelques-uns des nôtres. La gravure imprimée par Schöffer compte en tout cas comme la première représentation gravée de médecins, et sans doute d'herboristes par la même occasion.

Quant à la préface de l'ouvrage, elle est attribuée à Bernhard von Breidenbach, célèbre pour son voyage en Terre-Sainte.

Ce livre, d'abord manuscrit puis diffusé par l'imprimerie, eut un très rapide succès : dès le 22 août 1485 , à Augsbourg, paraît sans nom d'imprimeur (mais on sait qu'il s'agit de Hans Schœnsperger), une autre édition - on pourrait presque dire une contrefaçon 
si l'on osait cet anachronisme - comptant celle-ci 36 lignes. La gravure liminaire est elle aussi copiée, avec beaucoup de soin, mais dans un format plus réduit, et quelques différences y sont introduites : par exemple une pomme de pin est apparue dans l'écu qui surplombe l'assemblée des médecins. Il a fallu moins de cinq mois à cet atelier d'imprimerie pour réaliser cette copie, gravures comprises, ce qui est une performance. Notons que, comme c'est généralement le cas lorsqu'une gravure est copiée, le dessin est inversé en symétrie gauche-droite.

L'imprimeur strasbourgeois Johann Grüninger a produit de son côté une édition de cet ouvrage, appelée aussi " le petit Hortus sanitatis ", et c'est le feuillet liminaire de cette édition que la BNU a pu se procurer. Il mesure $26 \times 16,7 \mathrm{~cm}$. Les ouvrages spécialisés sur les livres anciens de sciences naturelles le connaissent bien. En Alsace, il est présent dans les collections de la bibliothèque municipale de Colmar, mais privé de son feuillet liminaire.

Johann Grüninger est le troisième en date des imprimeurs étrangers installés à Strasbourg. Il est né à Markgröningen, en Souabe, d'où provient son nom. Avant d'acheter le droit de bourgeoisie, le 2 octobre 1482, il travailla comme " maître " dans des ateliers d'imprimerie de Bâle, de Spire, de Reichenau et de Nuremberg. Il collaborait avec des imprimeurs-éditeurs d'Augsbourg et sans doute d'autres lieux de Souabe, et le document présenté ici en témoigne. Cependant, ce n'est qu'à partir de 1500 que ses talents dans la production de livres illustrés vont commencer à briller. Jusqu'à cette date, "ses " bois gravés sont d'une facture nettement inférieure à ce qui fera par la suite sa renommée. A l'époque où nous sommes, qui est celle des premières réalisations de son atelier, on se contente de copier les gravures parues dans les ouvrages qui ont trouvé le succès. Encore le fait-on avec plus ou moins de soin. Ce soin, on peut le mettre dans la beauté du dessin ou dans l'exactitude de la copie. C'est ce dernier choix, me semble-t-il, qu'a fait le " tailleur de formes" ("Formschneider") à qui nous devons ce frontispice. Notons que rien ne nous dit que les graveurs de l'une et de l'autre version ne sont pas la même personne ayant travaillé à deux versions très légèrement différentes du même motif pour deux imprimeurs différents. L'anonymat des artistes empêche d'en savoir plus à ce sujet.
Nous pourrions presque proposer ici un jeu des sept différences, entre deux gravures extrêmement proches, au trait près, et avec cette difficulté supplémentaire qu'il n'est pas sûr qu'il y ait sept différences : peut-être y en a-t-il plus, peut-être moins...

Pour enrichir cette rubrique, citons également un autre avatar de cette même gravure, issu de l'édition d'Augsbourg (Johann Schœnsperger, 1496) où au folio 3 verso figure une représentation de trois médecins, inspirée directement des gravures précédentes, mais où on les voit à présent assis dans une pharmacie. Le motif de l'assemblée des médecins va en effet disparaître des éditions du Hortus sanitatis entre 1487 et 1490, remplacé par la représentation d'un professeur à son pupitre ou par des savants observant des animaux qui les environnent. La comparaison avec les autres éditions de la même œuvre produites à la même époque est possible notamment grâce à la numérisation effectuée par la Bibliothèque de l'Etat de Bavière, qui en propose un grand nombre.

Ajoutons pour terminer que le célèbre ouvrage en 23 volumes d'A. Schramm, Der Bilderschmuck der Frühdrucke (Leipzig, 1920-1943), ne publie pas cette gravure de Grüninger, pour la raison suivante, qu'il exprime en page 9 $\mathrm{du}$ volume $\mathrm{XX}$ : il n'a pas réussi à en trouver un exemplaire non colorié. Il remet au volume terminal de son œuvre la reproduction de cette gravure, mais ce volume terminal n'a jamais paru. Les gravures représentant des plantes sont néanmoins présentes dans son répertoire des œuvres strasbourgeoises. Le volume de Grüninger compte, lorsqu'il est complet, 384 bois de plantes, animaux et minéraux, c'est-à-dire cinq de plus que l'original " copié ", l'édition de Mayence de Peter Schoeffer. Notons enfin qu'une autre copie, là encore inversée par rapport à celle de l'édition de Grüninger, figure dans un incunable dont on ignore le lieu d'origine et l'imprimeur, mais qu'on situe cependant à Strasbourg vers 1488-1494.

Cette acquisition porte à sept le nombre d'éditions incunables de l'Hortus sanitatis possédées (en entier ou en partie) par la BNU. Elle porte la cote K.2.056.

\section{Daniel Bornemann}




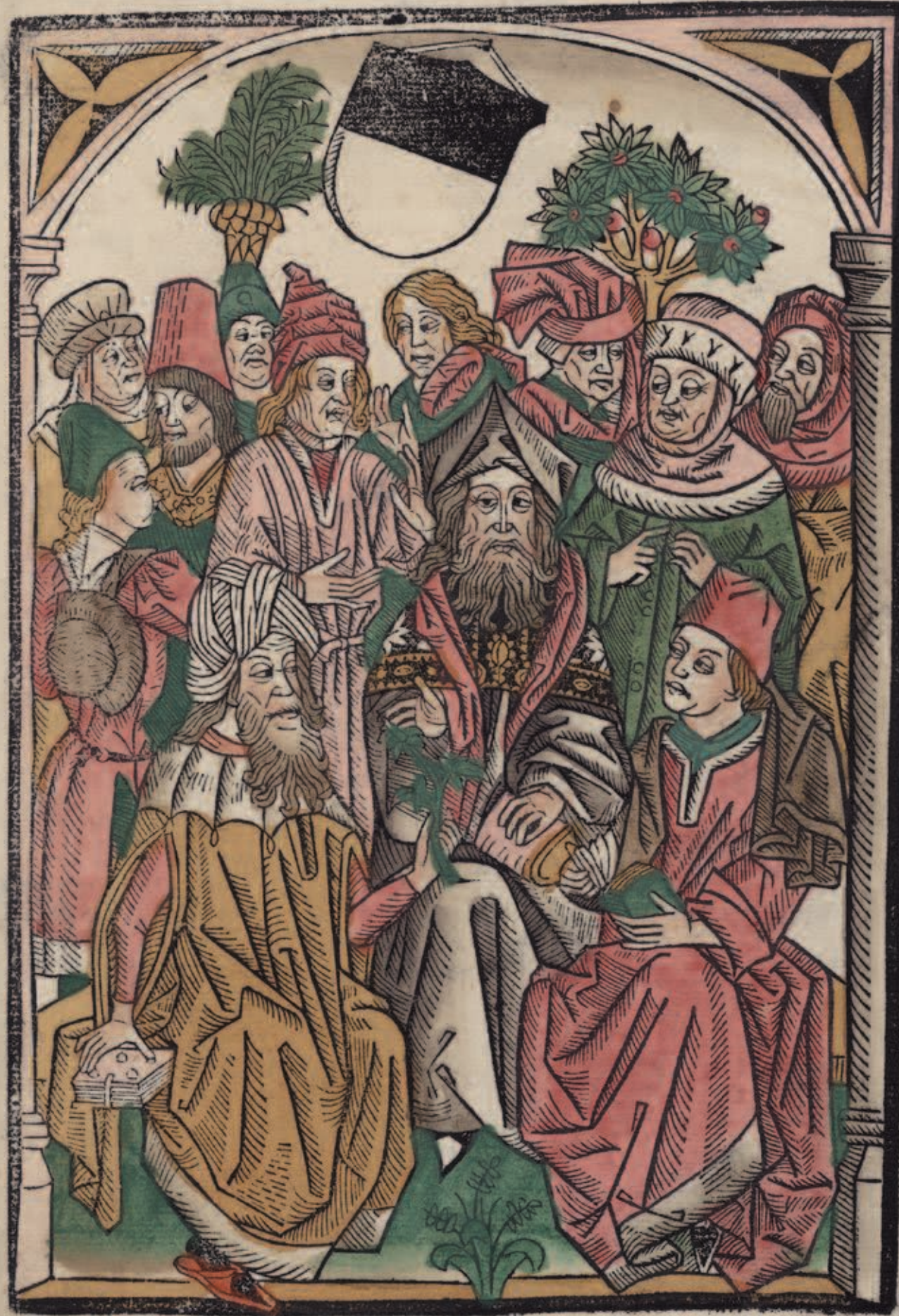

Verso du feuillet liminaire du Gart der Gesundheit ou Hortus sanitatis, de Johannes de Cuba, Ulm : Konrad Dinckmut, 31 mars 1487 (coll. BNU) 